\section{Large-scale production of single-walled carbon nanotubes by the electric-arc technique}

\section{Journet ${ }^{\star}$, W. K. Maser ${ }^{\star} \dagger$, P. Bernier ${ }^{\star}$, A. Loiseau $\ddagger$, M. Lamy de la Chapelle $₫$, S. Lefrant $\$$, P. Deniard $\$$, R. Lee \& J. E. Fischer\|}

* Groupe de Dynamique des Phases Condensées, Université de Montpellier II, 34095 Montpellier cedex 5, France

†LS, ONERA, BP 72, 92322 Châtillon cedex, France

$\$ I M N$, Université de Nantes, BP 32229, 44322 Nantes cedex 3, France \| LRSM, University of Pennsylvania, Philadelphia, Pennsylvania 19104-6272, USA

Single-walled carbon nanotubes (SWNTs) offer the prospect of both new fundamental science and useful (nano)technological applications ${ }^{1}$. High yields (70-90\%) of SWNTs close-packed in bundles can be produced by laser ablation of carbon targets ${ }^{2}$. The electric-arc technique used to generate fullerenes and multiwalled nanotubes is cheaper and easier to implement, but previously has led to only low yields of SWNTs ${ }^{3,4}$. Here we show that this technique can generate large quantities of SWNTs with similar characteristics to those obtained by laser ablation. This suggests that the (still unknown) growth mechanism for SWNTs must be independent of the details of the technique used to make them. The ready availability of large amounts of SWNTs, meanwhile, should make them much more accessible for further study.

In our electric arc-discharge apparatus ${ }^{5}$, the arc is generated between two electrodes in a reactor under a helium atmosphere (660 mbar). The cathode was a graphite rod $(16 \mathrm{~mm}$ diameter, $40 \mathrm{~mm}$ long) and the anode was also a graphite rod $(6 \mathrm{~mm}$ diameter, $100 \mathrm{~mm}$ long) in which a hole (3.5 mm diameter, $40 \mathrm{~mm}$ deep) had been drilled and filled with a mixture of a metallic catalyst and graphite powder. The arc discharge was created by a current of $100 \mathrm{~A}$; a voltage drop of $30 \mathrm{~V}$ between the electrodes was maintained by continuously translating the anode to keep a constant distance $(\sim 3 \mathrm{~mm})$ between it and the cathode. Typical synthesis times were $\sim 2 \mathrm{~min}$. As the catalyst we used mixtures such as $\mathrm{Ni}-\mathrm{Co}, \mathrm{Co}-\mathrm{Y}$ or $\mathrm{Ni}-\mathrm{Y}$ in various atomic percentages; these are known to yield a series of interesting carbon nanostructures ${ }^{6}$. The mixture used by Guo et al. ${ }^{7}$ during their laser ablation process (Co and $\mathrm{Ni}$, both at 0.6 at.\%) did not produce a good yield of nanotubes in our case. However, we found that a mixture of 1 at.\% Y and 4.2 at.\% Ni gave the best results. In this case we observed (in a total carbon mass of $2 \mathrm{~g}$ ): (1) large quantities of rubbery soot condensed on the chamber walls; (2) web-like structures between the cathode and the reactor walls (no webs when either Y or Ni were absent); (3) a cylindrical deposit at the cathode's end; and (4) a small 'collar' ( $\sim 20 \%$ of the total mass) around the cathode deposit, as a black, very light and porous but free-standing material.

Within all these products it was possible to observe by scanning electron microscopy (SEM; using a JEOL JSM 6300F instrument) filament-like structures that are more or less dense, depending on where in the reactor they were deposited. The 'collar' deposit was densest; the soot was the least dense. A characteristic SEM image of the collar deposit (Fig. 1) shows large amounts of entangled carbon filaments, homogeneously distributed over large areas (here at least a few square millimetres) and with diameters ranging from 10 to $20 \mathrm{~nm}$. The average length between two entanglement points is several micrometres; we could not identify any filament ends. From several SEM images we estimate the yield of these filaments (with respect to the total volume of the solid material) to be of the order of
$80 \%$. Higher magnification confirms the existence of entangled filaments although it is not possible at this stage to identify the nature of the crosslinks.

High-resolution transmission electron microscopy (HRTEM; using a JEOL 4000FX instrument) images of the collar deposit (Fig. 2) show that each filament consists of smaller aligned SWNTs self-organized into bundle-like crystallites with diameters ranging

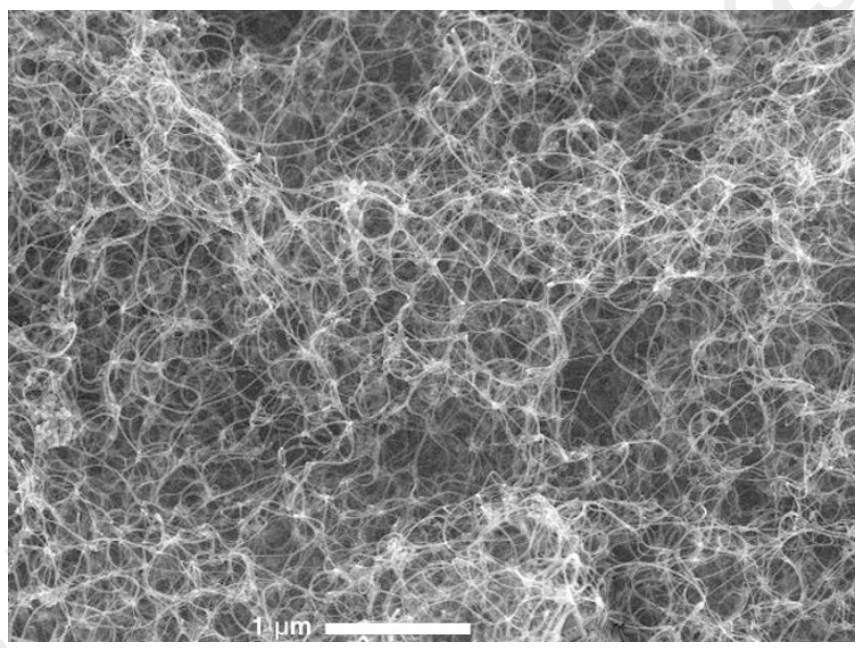

Figure 1 Scanning electron microscopy image of the light and porous material that formed as a collar around the cathode deposit in our apparatus, showing a high density of entangled carbon filaments. Scale bar, $1 \mu \mathrm{m}$.

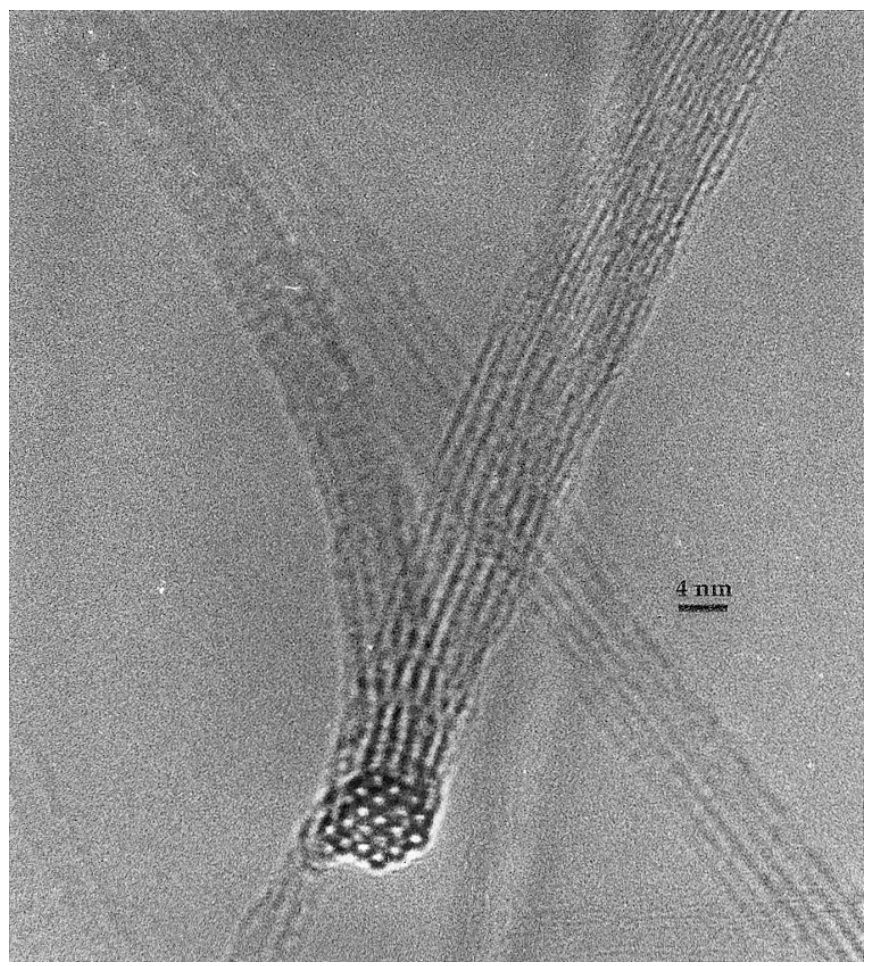

Figure 2 High resolution transmission electron microscopy view of one bundle from the collarette bent in such a way that it is seen edge-on. The bundle has a uniform width and consists of 20 SWNTs of almost uniform diameter, packed according to a triangular lattice. The focusing conditions are such that the lattice is imaged as a triangular arrangement of strong white dots (which are located at the centres of the SWNTs) on a dark background. Scale bar, $4 \mathrm{~nm}$. 
from 5 to $20 \mathrm{~nm}$. As these bundles are often bent, some portions are orientated parallel to the electron beam and are therefore seen edgeon (Fig. 2). The tube diameters are around $1.4 \mathrm{~nm}$, and the average distance between them is $1.7 \mathrm{~nm}$. Evidence for periodic packing is also given by electron diffraction patterns obtained from an assembly of bundles (not shown).

The number of tubes within a bundle is typically of the order of 20, and their section can be either roughly circular (Fig. 2) or elongated along a dense direction of the lattice. Larger-diameter bundles consist mostly of an assembly of smaller bundles separated by twin-like boundaries. The intra-bundle organization is also evidenced by the existence of diffraction fringes whose spacing is directly related to the lattice parameter and depends on the orientation of the crystalline bundle with respect to the electron beam.

Besides the bundles, we see randomly distributed spherical nanoparticles ranging from 3 to $20 \mathrm{~nm}$ in diameter, usually embedded in amorphous carbon. These nanoparticles probably consist of the remaining metallic catalyst. The SWNTs pass through

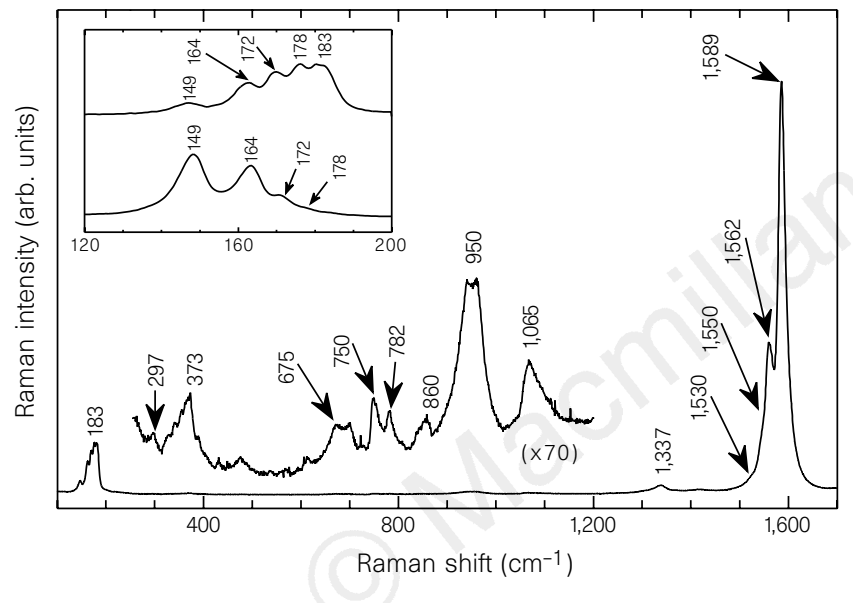

Figure 3 Raman spectrum of SWNTs recorded at room temperature, using an excitation wavelength of $514.5 \mathrm{~nm}$. The intensity in the frequency range between 250 and $1,200 \mathrm{~cm}^{-1}$ has been magnified 70 times. Inset, the low-frequency range (120-200 $\mathrm{cm}^{-1}$ ) recorded with high resolution at two different spots on the sample.

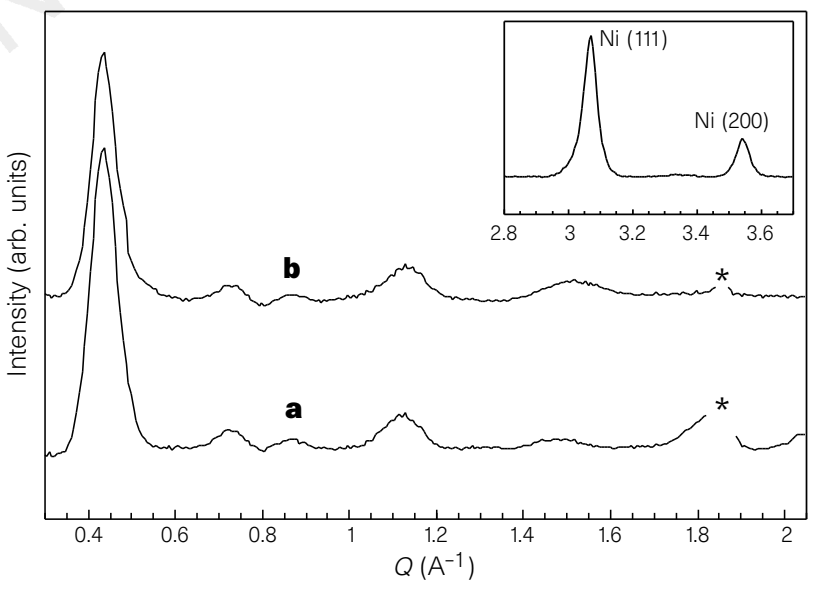

Figure $4 X$-ray diffraction patterns at low angle of our collar sample (trace a) and of the sample obtained by the laser ablation technique by Thess et al. ${ }^{2}$ (trace b). The graphite peak, due to remaining graphitic particles, has been removed for clarity; its position is shown by an asterisk. Inset, diffraction pattern associated with the Ni nanoparticles. them with no obvious evidence of a direct link. We have seen no other metallic or carbonaceous structures in the deposits.

It is difficult to determine the SWNT diameters accurately from electron microscopy, but the tube configuration can be studied in detail using Raman spectroscopy (Fig. 3). The spectrum exhibits three main zones at low $\left(140-200 \mathrm{~cm}^{-1}\right)$, intermediate (300$\left.1,300 \mathrm{~cm}^{-1}\right)$ and high $\left(1,500-1,600 \mathrm{~cm}^{-1}\right)$ frequencies, and is characteristic of SWNTs (other carbon materials produce other types of spectra $\left.^{8,9}\right)$. The high-frequency bands can be decomposed into two main peaks around 1,589 and $1,562 \mathrm{~cm}^{-1}$ with shoulders at 1,550 and $1,530 \mathrm{~cm}^{-1}$. These features have previously been assigned to a splitting of the $\mathrm{E}_{2 g}$ mode of graphite ${ }^{10}$. The very-high-resolution spectrum obtained in the low-frequency domain (Fig. 3 inset) shows at least five components at 149,164,172, 178 and $183 \mathrm{~cm}^{-1}$. These two spectra were recorded for exactly the same experimental conditions and sample, except that the laser beam explored two different areas. Thus the spectrum in this frequency domain is very sensitive to the sample area investigated, in contrast with that in the range 1,500$1,650 \mathrm{~cm}^{-1}$. In addition, different excitation wavelengths produce different relative intensities of these bands, indicating strong resonance behaviour. According to earlier calculations ${ }^{11}$, these modes are expected to be of $\mathrm{A}_{g}$ symmetry, and their frequency increases with decreasing tube diameter. Therefore our results clearly reflect a distribution in diameters (or of helical pitch for a given diameter). It can be shown theoretically ${ }^{10}$ that only 'armchair' tubes (with diameter and pitch parameters $n=m$ ranging from 6 to 12) generate Raman modes between 750 and $800 \mathrm{~cm}^{-1}$. In Fig. 3 the peaks in the frequency range $300-1,200 \mathrm{~cm}^{-1}$ can be mostly identified as overtones and combinations of lower-frequency modes; but the modes of 750 and $782 \mathrm{~cm}^{-1}$ are characteristic of such armchair nanotubes.

$\mathrm{X}$-ray diffraction was performed using an INEL CPS 120 detector in Debye-Scherrer geometry. A small flake from ground collar material was attached to an etched $\mathrm{Si}(111)$ wafer moistened with ethyl alcohol. After background subtraction, the data show an intense peak near diffusion vector $Q=0.43 \AA^{-1}$ and four weaker peaks up to $1.8 \AA^{-1}$ (Fig. 4 trace a). These low- $Q$ scattering peaks indicate the existence of a two-dimensional lattice of SWNTs organized in bundles ${ }^{2}$. The width of the peak at $Q=0.43 \AA^{-1}$ yields a coherence length of the order of $100 \AA$, in very good agreement with the average transverse size of the bundles. The region above $Q=3 \AA^{-1}$ (Fig. 4 inset) is dominated by two peaks at 3.1 and $3.6 \AA^{-1}$ which correspond to the (111) and (200) reflections of pure face-centred cubic nickel. The analysis of their shapes (using the Warren-Averbach ${ }^{12}$ method) results in a particle size distribution ranging from 2 to $21 \mathrm{~nm}$, with an average size of $11.2 \mathrm{~nm}$. Our $\mathrm{X}$-ray diffraction data are entirely consistent with the observations made by HRTEM.

A direct comparison of the X-ray diffraction data obtained by Thess et al. ${ }^{2}$ (Fig. 4 trace b) and by us reinforces the great similarity between both sample characteristics: yields in the range 70-90\%, diameters around $1.4 \mathrm{~nm}$, crystalline bundles of a few tens of tubes and only a few isolated SWNTs. Our results therefore imply that there is an unique growth mechanism for these nanotubes, which does not strongly depend on the details of the experimental conditions, but which depends much more on the kinetics of carbon condensation in a non-equilibrium situation. Temperature, and temperature gradients in space and time, must play an important role, as can be seen by the fact that most of the SWNTs were found in a very specific zone of the reactor (a few centimetres around the cathode). Additionally, the use of a second element beside the catalyst $\mathrm{Ni}$ or Co during the evaporation process is also critical. In our experiments it is yttrium that strongly favours the growth of SWNTs.

Our electric-arc technique is able to produce gram quantities of well-defined single-walled nanotubes in the form of highly crystalline bundles. It is a cheap method which could be scaled up easily, 
and so seems to be a very promising alternative to the double-laserablation technique ${ }^{2}$.

Received 18 April; accepted 17 June 1997

1. Dresselhaus, M. S., Dresselhaus, G. \& Eklund, P. C. Science of Fullerenes and Carbon Nanotubes (Academic, San Diego, 1996).

2. Thess, A. et al. Crystalline ropes of metallic carbon nanotubes. Science 273, 483-487 (1996).

3. Iijima, S. \& Ichihashi, T. Single-shell carbon nanotubes of 1-nm diameter. Nature 363, 603-605 (1993).

4. Bethune, D. S. et al. Cobalt-catalysed growth of carbon nanotubes with single-atomic-layer walls. Nature 363, 605-607 (1993).

5. Zahab, A. Synthesis, characterization and physical studies of fullerenes $\mathrm{C}_{60}$ and $\mathrm{C}_{70}$ and derivatives. Thesis, Univ. Montpellier II (1992).

6. Maser, W. K., Lambert, J. M., Ajayan, P. M., Stephan, O. \& Bernier, P. Role of Y-Ni-B mixtures in the formation of carbon nanotubes and encapsulation into carbon clusters. Synth. Metals 77, 243-247 (1996)

7. Guo, T., Nikolaev, P., Thess, A., Colbert, D. T. \& Smalley, R. E. Catalytic growth of single-walled nanotubes by laser vaporization. Chem. Phys. Lett. 243, 49-54 (1995).

8. Lamy de la Chapelle, M. et al. Elaboration and spectroscopic characterization of carbon nanostructures. Proc. SPIE 2854, 296-300 (1996).

9. Holden, J. M. et al. Raman scattering nanoscale carbons generated in a cobalt-catalyzed carbon plasma. Chem. Phys. Lett. 220, 186-191 (1994).

10. Eklund, P. C., Holden, J. M. \& Jishi, R. A. Vibrational modes of carbon nanotubes: spectroscopy and theory. Carbon 33, 959-972 (1995).

11. Rao, A. M. et al. Diameter-selective Raman scattering from vibrational modes in carbon nanotubes Science 275, 187-191 (1997).

12. Warren, B. E. \& Averbach, B. L. J. Appl. Phys. 21, 595-607 (1950).

Acknowledgements. The Montpellier and Nantes groups were supported by the European Community through its Training and Mobility of Researchers programme.

Correspondence and requests for materials should be addressed to P.B. (e-mail: pat@gdpc.univmontp2.fr).

\section{Enantioseparation using apoenzymes immobilized in a porous polymeric membrane}

\section{Brinda B. Lakshmi \& Charles R. Martin}

Department of Chemistry, Colorado State University, Fort Collins, Colorado 80523, USA

Chemical separations represent a large portion of the cost of bringing any new pharmaceutical product to the market. Membrane-based separation technologies ${ }^{1,2}$, in which the target molecule is selectively extracted and transported across a membrane, are potentially more economical and easier to implement than competing separations methods; but membranes with higher transport selectivities are required. Here we describe an approach for preparing highly selective membranes which involves immobilizing apoenzymes within a microporous composite. The apoenzyme selectively recognizes its substrate molecule and transports it across the composite membrane, without effecting the unwanted chemical conversion of the substrate molecule to product. We demonstrate this approach using three different apoenzymes. Most importantly, it can be used to make enantioselective membranes for chiral separations, one of the most challenging and important problems in bioseparations technology. We are able to achieve a fivefold difference between the transport rates of $\mathrm{D}$ - and $\mathrm{L}$-amino acids.

Membranes for chemical separations must recognize and transport a specific desired molecule and reject (or transport at lower rates) other molecules present with the desired molecule ${ }^{1,2}$. Living organisms have solved this problem by incorporating molecularrecognition proteins into lipid bilayer membranes ${ }^{3}$. By analogy, prototype membranes for chemical separations have been prepared by incorporating molecular-recognition proteins within synthetic membranes ${ }^{4,5}$. Enzymes might seem to be a likely choice because an enzyme selectively recognizes and binds its substrate molecule. However, enzymes also catalyse a chemical reaction on this molecule, and this unwanted chemical transformation must be subsequently undone in a following chemical $\operatorname{step}^{6-8}$. In addition, previous enzyme-based membranes for chemical separations have been of the unwieldy (and impractical) liquid-membrane design ${ }^{8}$. For these reasons, although enzymes are very useful in bioreactors $^{9,10}$, they have not proved useful as molecular-recognition agents in membranes for chemical separations.

It occurred to us that the unwanted chemical conversion of the substrate molecule ${ }^{8}$ in enzyme-based separation could be circumvented by using an apoenzyme as the molecular-recognition agent. Enzymes often require a cofactor to accomplish chemical conversion of the substrate molecule. We will show here that if the apoenzyme (no cofactor) is incorporated into a membrane, the membrane will selectively transport the substrate molecule without the unwanted chemical conversion of this molecule. We use the apoenzymes within a compact, solid-state composite membrane that requires no chemical derivatization of the enzyme.

The membrane design (Fig. 1) consists of a microporous polycarbonate filter that is sandwiched between two thin films of the polymer polypyrrole. The apoenzyme is physically trapped within the pores of the membrane by the polypyrrole films. Apoenzyme is also entrapped within the polypyrrole films. When this (initially dry) sandwich membrane is exposed to a buffer solution, water and electrolyte diffuse through the polypyrrole films and flood the pores. Polypyrrole was chosen as the surface layer material because it is highly permeable to small molecules (for example, the substrate for the enzyme) but does not pass proteins ${ }^{11}$. In addition, strongly adherent ultrathin polypyrrole films can be grown across the surfaces of the polycarbonate membrane using an electropolymerization method ${ }^{11}$.

The host membrane used for most studies was a $10-\mu m$-thick filter (Poretics, Livermore, CA, USA) with cylindrical, 400-nmdiameter pores (pore density $\left.1 \times 10^{8} \mathrm{~cm}^{-2}\right)^{11-14}$. The as-received membrane was cleaned by immersion in $\mathrm{CH}_{3} \mathrm{CN}$, and a gold film was then sputtered ${ }^{11}$ across one of its surfaces. This Au film is too thin $(30 \mathrm{~nm})$ to block the pores (Fig. 1), and is used to electropolymerize $^{11,15}$ a polypyrrole film (thickness $\sim 100 \mathrm{~nm}$ ) across the membrane surface. Plugs of polypyrrole are also deposited a small distance $(\sim 1 \mu \mathrm{m})$ into the pores (Fig. 1). An Au film was then sputtered across the other surface of the membrane. The pores in the membrane were then loaded with the desired apoenzyme by vacuum filtration of an apoenzyme solution through the membrane $e^{11}$. The apoenzyme solution is applied to the uncoated membrane face so that the solution can freely enter the pores. The uncoated Au film was then used to electropolymerize a polypyrrole film across this surface of the membrane, thus trapping the enzyme within the pores. The completed membrane was mounted between the two halves of a U-tube permeation cell ${ }^{12}$. The rate and selectivity of transport of various molecules from the feed half-cell through the membrane and into the permeant half-cell were investigated.

Membranes loaded with alcohol dehydrogenase apoenzyme (apo$\mathrm{ADH}$ ) serve to illustrate this new membrane concept. Figure $2 \mathrm{a}$

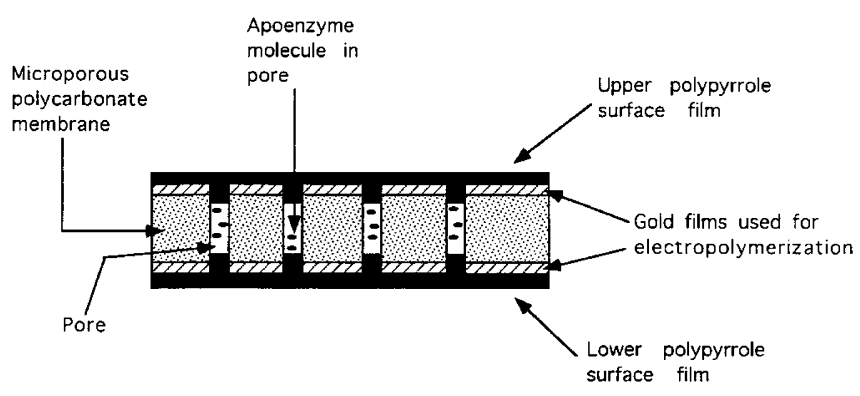

Figure 1 Schematic cross-section of the polypyrrole/polycarbonate/polypyrrole sandwich membrane with the apoenzyme entrapped in the pores. The membrane is drawn as coming out of the plane of the paper. The various components are not drawn to scale. 